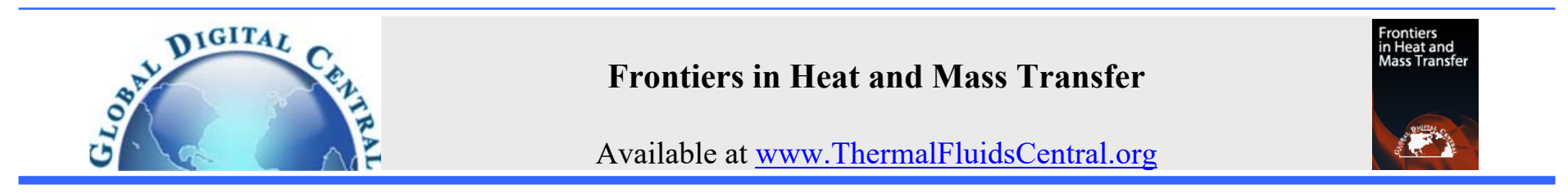

\title{
INDUCED MAGNETIC FIELD INTERACTION IN FREE CONVECTIVE HEAT ANDMASS TRANSFER FLOW OF A CHEMICALLY REACTIVE HEAT GENERATING FLUID WITH THERMO-DIFFUSION AND DIFFUSION-THERMO EFFECTS
}

\author{
Sanjib Sengupta ${ }^{\mathrm{a}, *}$ Amrit Karmakar ${ }^{\mathrm{b}}$ \\ ${ }^{a}$ Department of Mathematics, Assam University, Silchar, Assam, 788011, India \\ b Jawahar Navodaya Vidyalaya, R. C. Ghat, Khowai, Tripura, 799207, India
}

\begin{abstract}
An exact analysis is made to study the magnetohydrodynamics (MHD) free convective flow of an electrically conducting and chemically reacting Newtonian, incompressible, viscous fluid, flowing past an infinite vertical plate with combined heat and mass transfer. An inclined magnetic field of uniform strength is applied to the plate. As the value of the magnetic Reynolds number is of comparable order of magnitude, the effect of induced magnetic field is being considered and on the other hand due to weak voltage difference caused by the very low polarization charges, the influence of electric field is considered to be neglected. Cross-diffusion effects, like thermo-diffusion (Soret) and diffusion-thermo (Dufour) is being undertaken for this study due to high temperature and concentration gradients in the flow field. The influence of various physical parameters likes Hartmann number $(\mathrm{Ha})$, heat generation (source) parameter $(\mathrm{Qs})$, first order chemical reaction parameter $(F)$, Soret number $(\mathrm{Sr})$ and Dufour number $(\mathrm{Du})$ in particular is studied. The parametric effect of various pertinent parameter on the velocity, temperature and concentration profiles as well as on the induced, current density, total magnetic field as well as on the skin-friction, Nusselt number $\left(N u_{R}\right)$ and Sherwood number $\left(S h_{R}\right)$ are discussed. The increase in the magnetic field parameter (Hartmann number $(\mathrm{Ha})$ ) decreases the fluid velocity and current density but increases the skin-friction as well as induced magnetic field. The first order chemical reaction is found to decrease both the velocity field as well as induced magnetic field. The Soret number $(\mathrm{Sr})$ increases both the velocity field and induced magnetic field but Dufour number $(\mathrm{Du})$ only increases the velocity field while decreases the induced magnetic field.
\end{abstract}

Keywords: MHD free convection flow, induced magnetic field, heat generation, chemical reaction, Thermo-diffusion (Soret), Diffusion-thermo (Dufour)

\section{INRODUCTION}

Due to numerous applications of magnetohydro-dynamics (MHD) in geophysics and astrophysics, a lot of researchers have been given the due attention on it. Magnetohydrodynamics (MHD) is the study of motion of an electrically conducting fluid in the presence of magnetic field i.e. an electromagnetic field interacting with the velocity field of an electrically conducting fluid. On account of their varied importance, these flows have been studied by several authors. Ahmed et al. (2013) analyzed the MHD free convection mass transfer flow past an oscillating plate embedded in a porous medium with Soret effect. Sengupta and Karmakar (2016) studied the MHD mixed convection chemically reactive flow in radiative heat generating medium with Soret effect. Mamatha et al. (2015) carried out a study on thermal diffusion effect on MHD mixed convection unsteady flow of a micro polar fluid past a semi-infinite vertical porous plate with radiation and mass transfer. On the other hand an induced magnetic field modifies its original magnetic field as it generates its own magnetic field in the fluid, which modifies the motion of the fluids. Therefore in several physical situations it is required to consider. It is also become important due to its industrial applications in many devices and systems, like electric generator, magnetic induction, induction cooking, wireless energy transfer, induction welding, transformer etc. Kwanza and Balakiyema (2012) carried out a study on Magnetohydrodynamic free convective flow past an infinite vertical porous plate with magnetic induction. Ghosh et al. (2010) investigated the induced magnetic field effect on hydromagnetic free convection flow. Beg et al. (2009) study the induced magnetic field effect on non-similar, laminar, steady, electrically-conducting forced convection liquid metal boundary layer flow. Denno (1972) investigated the effect of induced magnetic field on the magnetohydrodynamic channel flow. Soundalgekar (1965) studied the hydro magnetic flow near an accelerated plate in the presence of a magnetic field, Sarveshanand and Singh (2015) also carried out a study on magnetohydrodynamic free convection flow between vertical parallel porous plates in the presence of induced magnetic field. Haque and Alam (2009) studied the transient heat and mass transfer by mixed convection flow from a vertical porous plate with induced magnetic field, constant heat and mass fluxes. Jha and Sani (2013) carried out a study on computational treatment of MHD of transient natural convection flow in a vertical channel due to symmetric heating in presence of induced magnetic field. Ahmed (2010) analyzed induced magnetic field with radiation fluid over a porous vertical plate. Chaudhary and Sharma (2006) investigated combined heat and mass transfer by laminar mixed convection flow from a vertical surface with induced magnetic field. Akbar et al. (2015) carried out study on influence of induced magnetic field and heat flux with the suspension of carbon nano tubes for the peristaltic flow in a permeable channel.

\footnotetext{
*Corresponding author.Email: sanjib_aus2009@rediffmail.com
} 
Kumar and Singh (2013) investigated unsteady MHD free convective flow past a semi-infinite vertical wall with induced magnetic field. Singh et al. (2010) carried out a study on hydromagnetic free convection in the presence of induced magnetic field. Nabil et al. (2015) studied the comparison between numerical and analytical solution for the motion of viscous fluid with heat and mass transfer through porous medium over a vertical infinite permeable plate in the presence of induced magnetic field. Raju et al. (2015) also carried out the study on radiation, inclined magnetic field and cross-diffusion effects on flow over a stretching surface. Sajid et al. (2015) carried out a study on modelling of two-dimensional MHD flow with induced magnetic field solution of peristaltic flow of a couple stress fluids in a channel. Sravanthi et al. (2013) analyzed thermo-diffusion and chemical reaction effects on a steady mixed convective heat and mass transfer flow with induced magnetic field.

Heat generation /absorption has an important role in many electronic devices, industries as well as nuclear reactor where heat is generated/absorbed which cause to minimize the longevity of the device. So due to importance of heat generation/ absorption in industrial and engineering field many researchers have work on its effect. Sengupta and Sen (2013) studied the free convective heat and mass transfer flow past an oscillating plate with heat generation, thermal radiation and thermo-diffusion effects. Muthtamilselvan et al (2014) also studied the effect of non-uniform heat generation on unsteady MHD flow over a vertical stretching surface with variable thermal conductivity. Venkataramana et al. (2017) carried out a study on the influence of heat generation (absorption) and thermal radiation on MHD laminar boundary layer flow over a moving cylindrical rod.

Chemical reaction is of great importance to the engineer and scientists due to its universal occurrence in many branches of science and engineering. A few field of interest where combined heat and mass transfer along with chemical reaction play an important role in chemical reaction process such as food processing and polymer production. Sengupta and Ahmed (2015) considered the study on chemical reaction effect on unsteady MHD free convective radiative flow past an oscillating plate embedded in porous media with thermal diffusion. SudheerBabu, and Satya Narayana (2009) studied the effects of the chemical reaction and radiation absorption on free convection flow through porous medium with variable suction in the presence of uniform magnetic field. Singh et al. (2016) studied the effect of inclined magnetic field on unsteady flow past a moving vertical plate with variable temperature. Sandeep and Sugunamma (2014) also analyzed radiation and inclined magnetic field effects on unsteady hydro magnetic free convection flow past an impulsively moving vertical plate in a porous medium. Sugunamma et al. (2013) also carried out a study on inclined magnetic field and chemical reaction effects on flow over a semi-infinite vertical porous plate through porous medium. Recently Ahmed et al. (2017) study the effects of chemical reaction, heat and mass transfer and viscous dissipation over a MHD flow in a vertical porous wall using perturbation method.

It is well known fact that the driving potential for the phenomenon of transport of mass is not alone concentration gradients, but high temperature gradients as well. The dual process by which mass gets transferred is known as thermo-diffusion or Soret effect. Bhavana (2013) considered the study on the Soret effect on free convective unsteady MHD flow over a vertical plate with heat source, Sengupta (2005) investigated Soret effect on unsteady heat and mass transfer flow of radiative chemically reactive fluid past an oscillating plate embedded in porous media. Raju et al. (2008) carried out the study on the Soret effects due to natural convection between heated inclined plates with magnetic field.

Again for transport of heat, the temperature gradient is not only the driving force, but a high concentration gradient is also a means for transport of heat. This process is known as Diffusion - thermo or Dufour effect. A lot of circumstances are there, where the temperature and concentration take place simultaneously this is when the Soret and Duffour effect is not neglected. Duffour effect is significant more in liquid mixture than in gases according to Platten and Legros (1984) Eckert and Drake (1972) also investigated the importance and applications of Dufour effect. With due importance of Dufour effect, several authors have contributed immensely on the combine effects of Soret and Dufour. Iinvestigators like Postelnicu (2004) carried out studied on the influence of a magnetic field on heat and mass transfer by natural convection from vertical surfaces in porous medium by considering Soret and Dufour effects. Reddy et al. (2016) considered the Soret and Dufour effects on MHD free convection flow of RivlinEricksen fluid past a semi-infinite vertical plate. Of late, Srinivasacharya et al. (2015) investigated the mixed convective heat and mass transfer flow along a vertical sinusoidal wavy surface in a porous medium with Soret and Dufour effects. Anghel et al. (2000) studied Soret and Dufour effects on free convection boundary layer flow over a vertical surface embedded in a porous medium. Very recently Sengupta and Ahmed (2016) investigated the problem of MHD free convective mass transfer flow of radiative uniform heat generation (absorption) fluid through a wavy permeable channel in presence of Soret and Dufour effects.

In spite of these studies, less attention has been given to the simultaneous effect of inclined and induced magnetic field on the prescribed flow situations. The objective of the present study is to do an exact analysis on a flow of an electrically conducting fluid of Newtonian, incompressible, viscous fluid with chemical reaction, Soret and Dufour effects in heat generating, Darcian porous media in presence of an inclined magnetic field, with due consideration of the influence of induced magnetic field.

\section{BASIC EQUATIONS AND ASSUMPTIONS}

\subsection{Basic Equations}

The vector forms of equations that describe the flow situation are as follows

The velocity vector and the magnetic field vectors are given by

$\vec{q}=(u(y), 0,0)$ and $\vec{B}=\left(B_{x}(y)-B_{0} \sin \alpha, B_{0} \cos \alpha, 0\right)$

The momentum equation gives

$\rho\left(\frac{\partial q}{\partial t}+(\vec{q} \cdot \vec{\nabla}) \vec{q}\right)=-\vec{\nabla} p+\mu \vec{\nabla}^{2} \vec{q}+\vec{J} \times \vec{B}$

Where the current density is

$\vec{J}=\vec{J}_{0}+\vec{J}_{1}$

The applied and induced current densities are respectively due to Ohm's and Ampere's laws as:

$\vec{J}_{0}=\sigma(\vec{q} \times \vec{B})$ and $\vec{J}_{1}=\frac{1}{\mu_{e}}\left(\vec{\nabla} \times \vec{B}_{1}\right) \sigma:$ Electrical

Conductivity

$\mu_{e}$ : Magnetic permeability

$\vec{B}_{1}=\left(B_{x}(y), 0,0\right)$ as the induced magnetic field.

The vector form of magnetic induction equation gives

$\lambda_{m} \vec{\nabla}^{2} \vec{B}-\{(\vec{q} \cdot \vec{\nabla}) \vec{B}-(\vec{B} . \vec{\nabla}) \vec{q}\}=0$ The vector form of energy

equation gives

$\rho C_{p}\left(\frac{\partial T}{\partial t}+(\vec{q} \cdot \vec{\nabla}) T\right)=k \vec{\nabla}^{2} T+Q^{*} \delta T+\frac{\rho D_{M} K_{T}}{C_{s}} \vec{\nabla}^{2} C$ The vector form of species

concentration equation gives

$\frac{\partial C}{\partial t}+(\vec{q} \cdot \vec{\nabla}) C=D_{M} \vec{\nabla}^{2} C+D_{T} \vec{\nabla}^{2} T-K_{l} \delta C+\frac{D_{M} K_{T}}{T_{M}} \vec{\nabla}^{2} T$

The other symbols are specified in the Nomenclature.

\subsection{Basic Assumptions}

The fundamental assumptions considered for the study are as follows: a) All the fluid properties except possibly the pressure are independent of variations of $x^{*}$-scale. 
b) The strength of the applied magnetic field, which is inclined to the plate, considered as constant. The induced as well as total magnetic field are consider for this study

c) As the applied voltage at the ends of the plate is weak as such polarization of charges is neglected, so the electric field is not considered for the study.

d) Due to moderate strength of the magnetic field, the viscous dissipation, as well as Joule heating is not considered for the study. e) Due to higher temperature gradient and concentration gradient, the cross diffusion effects, like thermo-diffusion (Soret) and diffusion-thermo (Dufour) have been considered for the study.

f) The plate is considered to be magnetically insulated.

g) The temperature as well as concentration of fluid particles near the plate surface is supposed to be more than their respective components at the free stream region.

\section{MATHEMATICAL FORMULATIONS OF THE PROBLEM AND SOLUTIONS}

A co-ordinate system $\left(x^{*}, y^{*}, z^{*}\right)$ has been introduced, with its $x^{*}$-axis along the length of the plate in the upward vertical direction, $\mathrm{y}^{*}$-axis taken perpendicular to the plate towards the fluid region and $\mathrm{z}^{*}$-axis considered along the width of the plate. A uniform magnetic field of strength $\vec{B}_{0}$ is applied inclined to the plate. An MHD fluid model has been developed in terms of a system of coupled partial differential equations combined with initio-boundary conditions as:

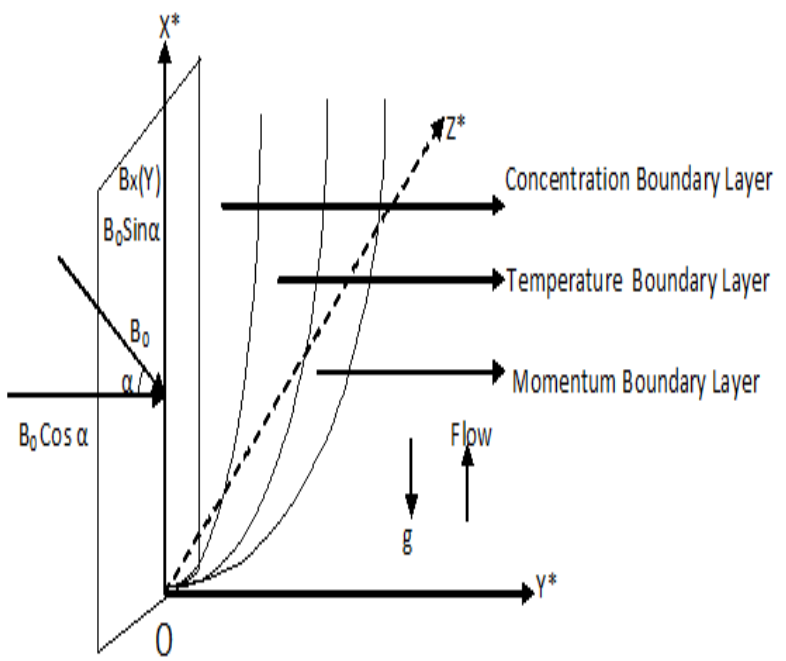

Fig.1. A Schematic representation of the flow configuration and geometry.

\section{$\mathbf{x}^{*}$ - momentum equation:}

$$
\begin{aligned}
& v \frac{\partial^{2} u^{*}}{\partial y^{* 2}}-\frac{\sigma}{\rho}\left(B_{0} \cos \alpha\right)^{2} u^{*}+\frac{B_{0} \cos \alpha}{\rho \mu_{e}} \frac{\partial B_{x}{ }^{*}}{\partial y^{*}}+g B^{*}{ }_{T}\left(T^{*}-T_{\infty}^{*}\right) \\
& +g B^{*}{ }_{c}\left(C^{*}-C_{\infty}^{*}\right)=0
\end{aligned}
$$

\section{Magnetic field induction equation:}

$$
\lambda_{m} \frac{\partial^{2} B_{x}^{*}}{\partial y^{* 2}}+B_{0} \cos \alpha \frac{\partial u^{*}}{\partial y^{*}}=0
$$

\section{The energy equation:}

$$
\frac{k}{\rho C_{p}} \frac{\partial^{2} T^{*}}{\partial y^{* 2}}+\frac{D_{M} K_{T}}{C_{S} C_{p}} \frac{\partial^{2} C^{*}}{\partial y^{* 2}}+\frac{Q_{s}^{*}}{\rho C_{P}}\left(T^{*}-T_{\alpha}^{*}\right)=0
$$

$D_{M} \frac{\partial^{2} C}{\partial y^{2}}+\frac{D_{M} K_{T}}{T} \frac{\partial^{2} T}{\partial y^{2}}-K_{i}\left(C^{*}-C_{\infty}^{*}\right)=0$

Subject to the into-boundary condition as;

$\left.\begin{array}{l}u^{*}(\mathrm{y})=0, \mathrm{~B}_{x}^{*}=0, T^{*}=T_{\infty}^{*}, C^{*}=C_{\infty}^{*} \text { for all } y^{*} \\ u^{*}(y)=0, \frac{\partial B_{x}^{*}}{\partial y^{*}}=0, \frac{\partial T^{*}}{\partial y^{*}}=-\frac{q}{k}, C^{*}=C_{w}^{*}, \text { for } y^{*}=0 \\ u^{*}(y) \rightarrow u_{\infty}^{*}, B_{x} \rightarrow 0, T^{*} \rightarrow T_{\infty} \& C^{*} \rightarrow C_{\infty}^{*} \text { for } y^{*} \rightarrow \infty\end{array}\right\}$

We consider the following set of non-dimensional quantities as:

$u=\frac{u^{*}}{U_{m}}, y=y^{*} U_{m}, B_{x}=\frac{B_{x}^{*}}{\sigma \mu_{e} B_{0}}, Q=\frac{K U_{m}}{q}\left(T^{*}-T^{*} \infty\right)$

$H a=\frac{B_{0}}{U_{m}} \sqrt{\frac{\sigma}{\rho v}}, G r=\frac{g \beta_{T} T_{\infty}^{*} q}{K v U_{m}^{3}}, G m=\frac{g \beta_{c}\left(C_{w}^{*}-C_{\infty}^{*}\right)}{v U_{m}^{3}}$

$\phi=\frac{C^{*}-C_{\infty}^{*}}{C_{w}^{*}-C_{\infty}^{*}}, J_{z}=\frac{J_{z}^{*}}{U_{m} B_{0}}, \mathrm{~F}=\frac{K l}{D_{M} U_{m}^{2}}, S r=\frac{q K_{T}}{K T_{m}\left(c_{w}^{*}-c_{\infty}^{*}\right) U_{m}}$

$\mathrm{Qs}=\frac{Q s^{*}}{U_{m}^{2} K}, D u=\frac{D_{M} K_{T}\left(C_{w}^{*}-C_{\infty}^{*}\right) U_{m} \rho}{q}$

The corresponding non-dimensional form of equations (1), (2), (3) and (4) are

$$
\begin{aligned}
& \frac{\partial^{2} u}{\partial y^{2}}-H a^{2} \cos ^{2} \alpha u+H a^{2} \cos \alpha \frac{\partial B_{x}}{\partial y}+G r \theta+G m \phi=0 \\
& \frac{\partial^{2} B_{x}}{\partial y^{2}}+\cos \alpha \frac{\partial u}{\partial y}=0 \\
& \frac{\partial^{2} \theta}{\partial y^{2}}+D u \frac{\partial^{2} \phi}{\partial y^{2}}+Q s \theta=0 \\
& \frac{\partial^{2} \phi}{\partial y^{2}}+S r \frac{\partial^{2} \theta}{\partial y^{2}}-F \phi=0
\end{aligned}
$$

With non-dimensional boundary conditions expressed as

$$
\left.\begin{array}{l}
u(\mathrm{y})=0, \mathrm{~B}_{x}=0, \theta=0, \phi=0 \text { for all } y \\
u(y)=0, \frac{\partial B_{x}}{\partial y}=0, \theta=-1, \phi=1 \text { at } \mathrm{y}=0 \\
u(y) \rightarrow 0, B_{x} \rightarrow 0, \theta \rightarrow 0, \& \phi \rightarrow 0 \text { for } y \rightarrow \infty
\end{array}\right\}
$$

On using straight forward integration scheme, the exact closed form of solutions for temperature, concentration, axial velocity and induced magnetic field are finally obtained as:

$$
\begin{aligned}
\theta(\mathrm{y})= & \frac{\xi_{1}^{2}\left(D u \xi_{2}^{3}+\xi_{2}^{2}+Q s\right)}{\xi_{1}^{3}\left(\xi_{2}^{2}+Q s\right)-\xi_{2}^{3}\left(\xi_{1}^{2}+Q s\right)} \exp \left(-\xi_{1} y\right) \\
& -\frac{\xi_{2}^{2}\left(D u \xi_{1}^{3}+\xi_{1}^{2}+Q s\right)}{\xi_{1}^{3}\left(\xi_{2}^{2}+Q s\right)-\xi_{2}^{3}\left(\xi_{1}^{2}+Q s\right)} \exp \left(-\xi_{2} y\right)
\end{aligned}
$$

The species concentration equation: 


$$
\begin{aligned}
\phi(\mathrm{y})= & \frac{-\left(\xi_{1}^{2}+Q s\right)\left(D u \xi_{2}^{3}+\xi_{2}^{2}+Q s\right)}{D u\left[\xi_{1}^{3}\left(\xi_{2}^{2}+Q s\right)-\xi_{2}^{3}\left(\xi_{1}^{2}+Q s\right)\right]} \exp \left(-\xi_{1} y\right) \\
& +\frac{\left(\xi_{2}^{2}+Q s\right)\left(D u \xi_{1}^{3}+\xi_{1}^{2}+Q s\right)}{D u\left[\xi_{1}^{3}\left(\xi_{2}^{2}+Q s\right)-\xi_{2}^{3}\left(\xi_{1}^{2}+Q s\right)\right]} \exp \left(-\xi_{2} y\right) \\
\mathrm{u}(\mathrm{y})= & \left(\frac{G r \psi_{1}+G m \psi_{3}}{\xi_{1}^{2}-2 H a^{2} \cos ^{2} \alpha}+\frac{G r \psi_{2}+G m \psi_{4}}{\xi_{2}^{2}-2 H a^{2} \cos ^{2} \alpha}\right) \exp \left(-\xi_{3} y\right) \\
& -\frac{G r \psi_{1}+G m \psi_{3}}{\xi_{1}^{2}-2 H a^{2} \cos ^{2} \alpha} \exp \left(-x_{1} y\right)-\frac{G r \psi_{2}+G m \psi_{4}}{\xi_{2}^{2}-2 H a^{2} \cos ^{2} \alpha} \exp \left(-\xi_{2} y\right) \\
B_{x}= & {\left[\begin{array}{c}
\frac{G r \psi_{1}+G m \psi_{3}}{x_{1}^{2}-2 H a^{2} \cos ^{2} \alpha} \\
\xi_{3}\left(\begin{array}{l}
G r \psi_{2}+G m \psi_{4} \\
\xi_{2}^{2}-2 H a^{2} \cos ^{2} \alpha
\end{array}\right) \exp \left(-\xi_{3} y\right) \\
-\frac{1}{\xi_{1}}\left(\frac{G r \psi_{1}+G m \psi_{3}}{\xi_{1}^{2}-2 H a^{2} \cos ^{2} \alpha}\right) \exp \left(-\xi_{1} y\right) \\
-\frac{1}{\xi_{2}}\left(\frac{G r \psi_{2}+G m \psi_{4}}{\xi_{2}^{2}-2 H a^{2} \cos ^{2} \alpha}\right) \exp \left(-\xi_{2} y\right)
\end{array}\right] \cos \alpha }
\end{aligned}
$$

Where

$$
\begin{aligned}
& \xi_{1}=\sqrt{\frac{(F-Q s)+\sqrt{(F-Q s)^{2}+4 F Q s(1-D u S r)}}{2(1-D u S r)}} \\
& \xi_{2}=\sqrt{\frac{(F-Q s)-\sqrt{(F-Q s)^{2}+4 F Q s(1-D u S r)}}{2(1-D u S r)}} \\
& \xi_{3}=\sqrt{2} H a \cos \alpha \\
& \psi_{1}=\frac{\xi_{1}^{2}\left(D u \xi_{2}^{3}+\xi_{2}^{2}+Q s\right)}{\xi_{1}^{3}\left(\xi_{2}^{2}+Q s\right)-\xi_{2}^{3}\left(\xi_{1}^{2}+Q s\right)} \\
& \psi_{2}=\frac{-\xi_{2}^{2}\left(D u \xi_{1}^{3}+\xi_{1}^{2}+Q s\right)}{\xi_{1}^{3}\left(\xi_{2}^{2}+Q s\right)-\xi_{2}^{3}\left(\xi_{1}^{2}+Q s\right)} \\
& \psi_{3}=\frac{-\left(\xi_{1}^{2}+Q s\right)\left(D u \xi_{2}^{3}+\xi_{2}^{2}+Q s\right)}{D u\left[\xi_{1}^{3}\left(\xi_{2}^{2}+Q s\right)-\xi_{2}^{3}\left(\xi_{1}^{2}+Q s\right)\right]} \\
& \psi_{4}=\frac{\left(\xi_{2}^{2}+Q s\right)\left(D u \xi_{1}^{3}+\xi_{1}^{2}+Q s\right)}{D u\left[\xi_{1}^{3}\left(\xi_{2}^{2}+Q s\right)-\xi_{2}^{3}\left(\xi_{1}^{2}+Q s\right)\right]}
\end{aligned}
$$

\section{SOME SIGNIFICANT QUANTITIES OF ENGINEERING INTEREST}

\subsection{Skin-friction at the plate}

The real part of the non-dimensional skin-friction coefficient at the plate is obtained as:

$$
\begin{aligned}
\tau_{\mathrm{R}}=-\left(\frac{\partial u}{\partial y}\right)_{y=0}= & \xi_{3}\left(\frac{G r \psi_{1}+G m \psi_{3}}{\xi_{1}^{2}-2 H a^{2} \cos ^{2} \alpha}+\frac{G r \psi_{2}+G m \psi_{4}}{\xi_{2}^{2}-2 H a^{2} \cos ^{2} \alpha}\right) \\
& -\xi_{1}\left(\frac{G r \psi_{1}+G m \psi_{3}}{\xi_{1}^{2}-2 H a^{2} \cos ^{2} \alpha}\right) \\
& -\xi_{2}\left(\frac{G r \psi_{2}+G m \psi_{4}}{\xi_{2}^{2}-2 H a^{2} \cos ^{2} \alpha}\right)
\end{aligned}
$$

\subsection{Rate of heat transfer coefficient}

The real part of the rate of heat transfer coefficient in terms of the Nusselt number is given as

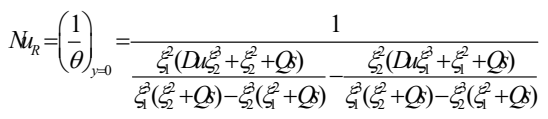

\subsection{Rate of mass transfer coefficient}

The real part of the mass transfer coefficient in terms of the Sherwood number is

$$
\begin{aligned}
& S h_{R}=-\left(\frac{\partial \phi_{R}}{\partial y}\right)_{y=0}=\frac{\xi_{2}\left(\xi_{2}^{2}+Q s\right)\left(D u \xi_{1}^{3}+\xi_{1}^{2}+Q s\right)}{D u\left[\xi_{1}^{3}\left(\xi_{2}^{2}+Q s\right)-\xi_{2}^{3}\left(\xi_{1}^{2}+Q s\right)\right]} \\
& -\frac{\xi_{1}\left(\xi_{1}^{2}+Q s\right)\left(D u \xi_{2}^{3}+\xi_{2}^{2}+Q s\right)}{D u\left[\xi_{1}^{3}\left(\xi_{2}^{2}+Q s\right)-\xi_{2}^{3}\left(\xi_{1}^{2}+Q s\right)\right]}
\end{aligned}
$$

\subsection{Current density and Total magnetic field}

The dimensional form of current density is given by

$$
J_{z}^{*}=u^{*} B_{0} \cos \alpha-\lambda_{m} \frac{\partial B_{x}^{*}}{\partial y^{*}}
$$

The corresponding non-dimensional form is

$$
\begin{aligned}
& J_{z}=u \cos \alpha-\frac{\partial B_{x}}{\partial y} \\
& J_{z}=2\left[\begin{array}{l}
\left(\begin{array}{c}
\frac{G r \psi_{1}+G m \psi_{3}}{\xi_{1}^{2}-2 H a^{2} \cos ^{2} \alpha} \\
+\frac{G r \psi_{2}+G m \psi_{4}}{\xi_{2}^{2}-2 H a^{2} \cos ^{2} \alpha}
\end{array}\right) \exp \left(-\xi_{3} y\right) \\
-\frac{G r \psi_{1}+G m \psi_{3}}{\xi_{1}^{2}-2 H a^{2} \cos ^{2} \alpha} \exp \left(-\xi_{1} y\right) \\
-\frac{G r \psi_{2}+G m \psi_{4}}{\xi_{2}^{2}-2 H a^{2} \cos ^{2} \alpha} \exp \left(-\xi_{2} y\right)
\end{array}\right] \cos \alpha
\end{aligned}
$$

The dimensional form of total magnetic field is found as,

$$
B=\sqrt{\frac{B_{x}^{*}}{\sigma \mu_{e} B_{0}}\left(\frac{B_{x}^{*}}{\sigma \mu_{e} B_{0}}-2 B_{0} \operatorname{Sin} \alpha\right)+B_{0}}
$$

The same in non-dimensional form is

$$
B=\sqrt{B_{x}\left(B_{x}-2 B_{0} \operatorname{Sin} \alpha\right)+B_{0}}
$$

\section{RESULTS AND DISCUSSION}

To investigate the influence of various physical parameters on the flow variables, numerical simulations have been made by assigning appropriate physical values of the governing parameters such as heat source parameter $(Q s)$, Hartmann number $(\mathrm{Ha})$, chemical reaction parameter $(F)$, Soret number $(S r)$, Dufour number $(D u)$, Thermal Grashof number $(\mathrm{Gr})$ and solutal Grashof number $(\mathrm{Gm})$.

Fig.2 illustrate the effect of heat source parameter $(Q s)$ and chemical reaction $(F)$ on a non- dimensional temperature profile $\left(\Theta_{R}, y\right)$ for a set of fixed value of chemical reaction parameter $(\mathrm{F}=1.0)$, heat source 
parameter $(\mathrm{Qs}=2.5)$, Dufour number $(D u=0.2)$ and Soret number $(S r=1.0)$. In fig. 2(a) the parametric increase in values of $Q_{s}$ is found to increase the temperature of fluid particles near the plate surface, this is due to the fact that, the temperature fluxes inside the thermal boundary layer get rises results of which, the values of $\theta_{R}$ increases but decreases gradually to its limiting value as normal distances increase. The effect of chemical reaction on the temperature profile $\left(\theta_{R}, y\right)$ is shown in figure 2(b). It is observed that the chemical reaction parameter decreases the temperature against normal distance. Due to increase in chemical reaction parameter, some amount of heat is absorbed by the medium to complete the process. This result in minimizing the thermal boundary layer thickness which is thus decreases the temperature near the plate.

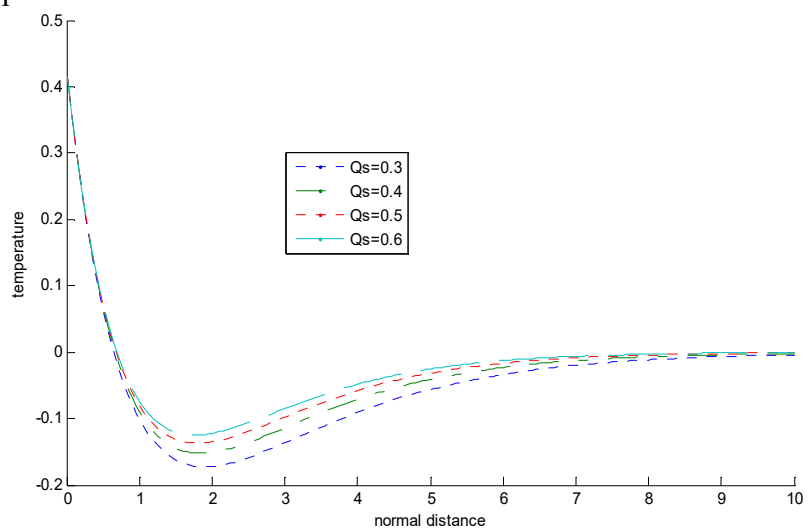

Fig. 2 (a) Temperature $\left(\theta_{R}\right)$ versus Normal distance (y) for arbitrary change in values of Heat source parameter $(Q s)$.

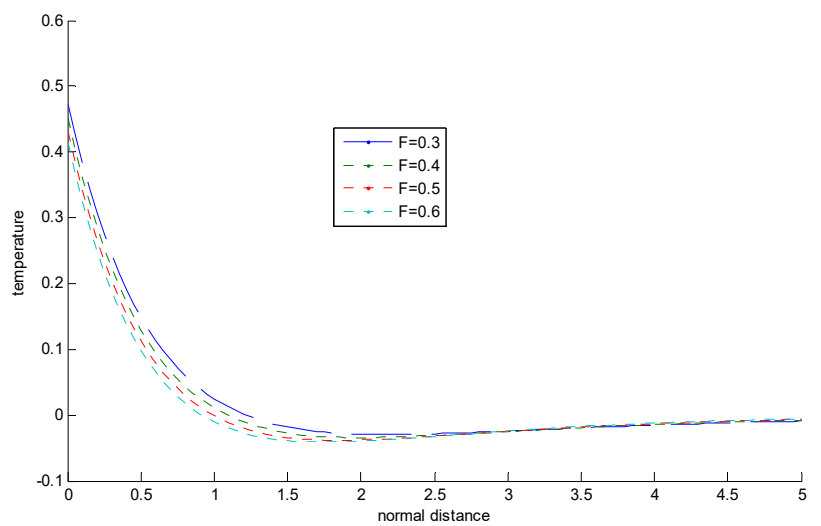

Fig. 2 (b) Temperature $\left(\theta_{R}\right)$ versus Normal distance $(y)$ for arbitrary change in values of Chemical reaction parameter $(F)$.

The effect of parameters like Soret number $(S r)$, Dufour number $(D u)$, heat source parameter $(Q s)$ and chemical reaction parameter $(F)$ on the concentration profiles $\left(\phi_{R}, \mathrm{y}\right)$ has been depicted graphically in fig. 3 for a set of fixed values of $F=1.0, Q s=0.2, S r=1.0$ (fig. 3(a), 3(c), 3(d)), $F=0.3, Q s=0.02, D u=3.9$ (fig.3(b)) and $D u=0.2$ (fig.3(c), 3(d)). It is observed that the concentration of fluid particles near the plate decreases due to increase in values of Dufour number $(D u)$, heat source parameter $(Q s)$ and chemical reaction parameter $(F)$, while decreasing due to increase in values of Soret number (Sr). In fig.3 (a) it is perceive that, as the values of Dufour number $(D u)$ increases within the range $0.5 \leq D u \leq 2.0$, the concentrationfluxes within the boundary layer increases though no significant effect is observed due to $D u \in[0,0.49]$ (approx.). This in turn increases concentration inside the solutal boundary layer. On the other hand due to increase in Soret number, the concentration gradient rises by the presence of large temperature gradient, which thus increases the concentration near the plate and is clearly observed in fig.3 (b).

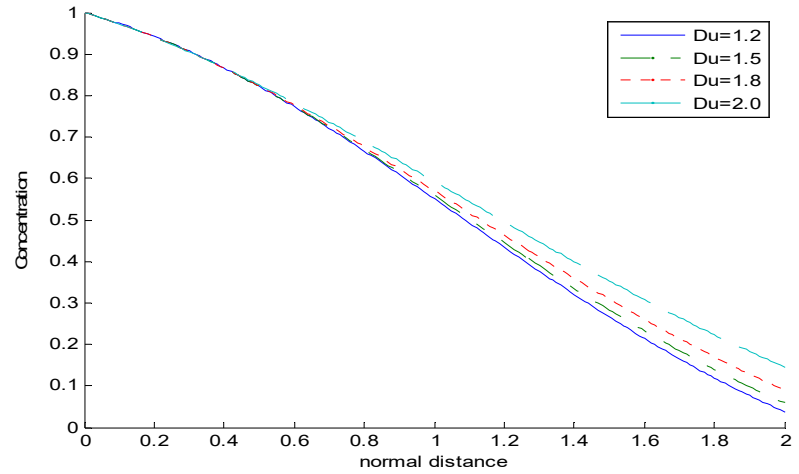

Fig. 3 (a) Concentration ( $\phi_{R}$ ) versus Normal distance (y) for arbitrary change in values of Dufour number $(D u)$.

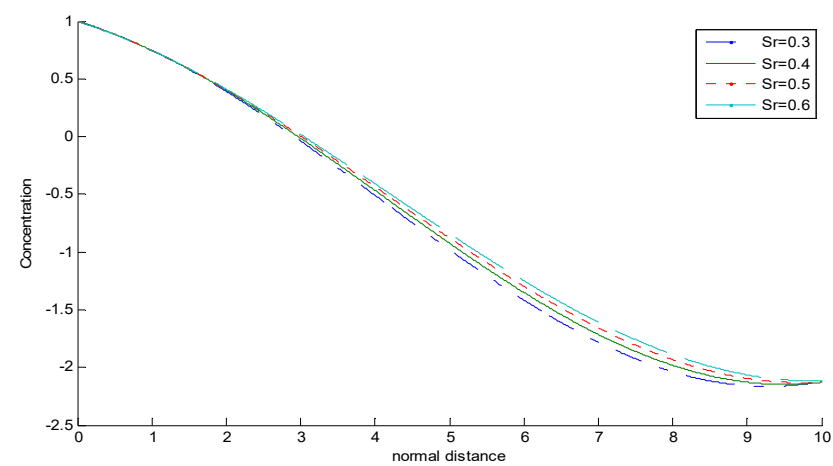

Fig. 3 (b) Concentration ( $\phi_{R}$ ) versus Normal distance (y) for arbitrary change in values of Soret number $(S r)$.

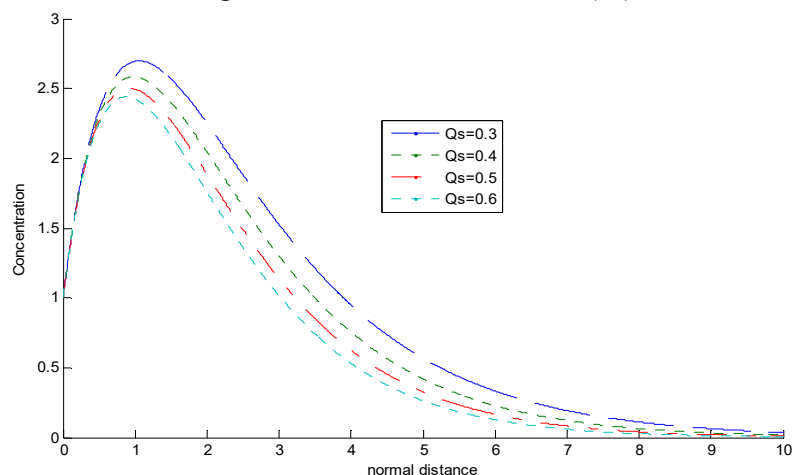

Fig. 3 (c) Concentration $\left(\phi_{R}\right)$ versus Normal distance (y) for arbitrary change in values of Heat source parameter $(Q s)$.

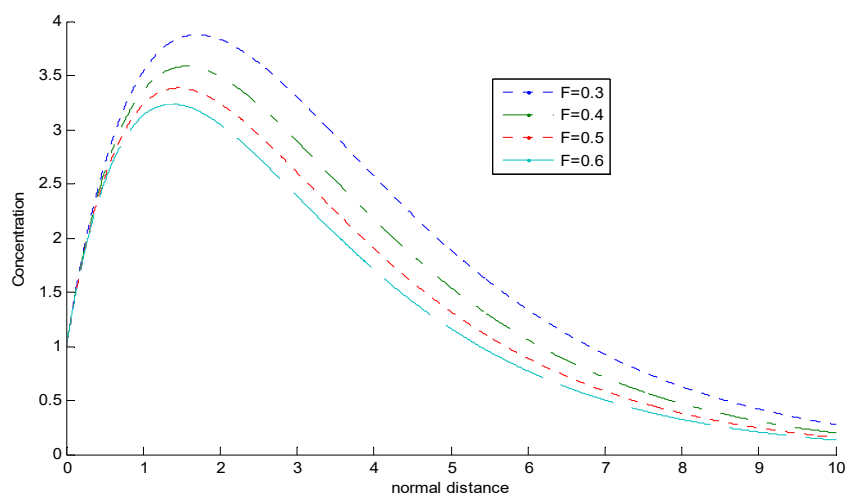

Fig. 3 (d) Concentration ( $\phi_{R}$ ) versus Normal distance (y) for arbitrary change in values of Chemical reaction parameter $(F)$. 
Due to raise in heat source parameter, the temperature gradient near the plate surface raises. This causes the density of the constituents to decrease which result in dropping the concentration near the plate surface as observed in fig.3(c). As observed in fig. 3(d), the fluid molecules from the higher concentration zone (plate region) transit towards lower concentration zone (free stream region) due to the enhancement of the first order chemical reaction as such the concentration declining due to increase in chemical reaction parameter.

Figure 4 show the influence of parameters like heat source parameter $(Q s)$, Soret number $(\mathrm{Sr})$ and Dufour number $(\mathrm{Du})$ on the velocity profile $\left(u_{R}, y\right)$ (fig. $\left.4(\mathrm{a})\right)$ and on the velocity profile $\left(u_{R}, Q s,\right)$ (fig. 4(b),4(c)) respectively for a set of fixed assigned values of $H a=3.0$ (fig.4(a), and 4(b), $\alpha=1.0476$ (fig. 4(a), 4(b), and 4(c), $F=1.0$, $D u=0.2, S r=1.0, G r=0.02, G m=0.02$ (fig. 4(a)) $F=4.0, \mathrm{y}=0.1, D u=0.01$, $G r=1.1, G m=1.0$ (fig. 4(b)), $F=1.5, y=0.0, S r=1.8, H a=0.5, G r=0.2$, $G m=0.2$ (fig.4(c)) It can be observed from figure 4(a) that, the fluid velocity increases due to increase in values of heat source parameter $(Q s)$. The presence of heat source parameter $(Q s)$ is found to increase the temperature of fluid particles (as observed in figure 1(a)) thus increases the kinetic energy of the fluid particles followed by increase in rate of flow, which thus increases the velocity of the flow. Again the influence of Soret $(\mathrm{Sr})$ and Dufour $(\mathrm{Du})$ numbers on the fluid velocity are depicted in fig. 4(b) and fig. 4(c) respectively. Due to the influence of Soret $(S r)$ and Dufour $(D u)$ numbers, the fluid velocity is found increasing. As the values of Soret $(S r)$ number enhance the concentration of fluid particles, the mass bouncy force due to species concentration accelerates followed by increase in fluid velocity. It is also observed that the fluid velocity achieving the maximum peak at approximately at $Q s=2$ thereafter for $2 \leq Q s \leq 10$, the fluid velocity declining gradually. Again in fig.4 (b) the velocity is found increasing due to increase in values of Dufour number $(D u)$. As the Dufour number increases the thermal bouncy force rises which thus accelerates the flow rate followed by increase in values of fluid velocity. It is also observed that the velocity takes its maximum pick in presence of Dufour number, for the values of $Q s=0.5$ (approx) and thereafter gradually decreases for $0.5 \leq Q s \leq 5$.

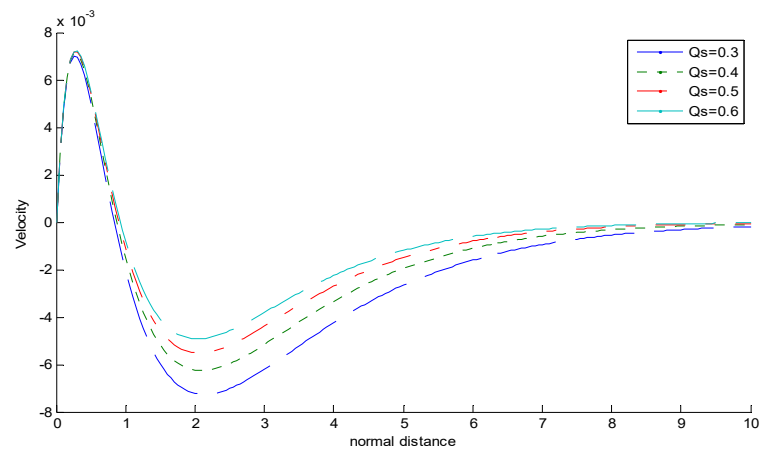

Fig. 4 (a) Velocity $\left(u_{R}\right)$ versus Normal distance $(y)$ for arbitrary change in values of Heat source parameter $(Q s)$

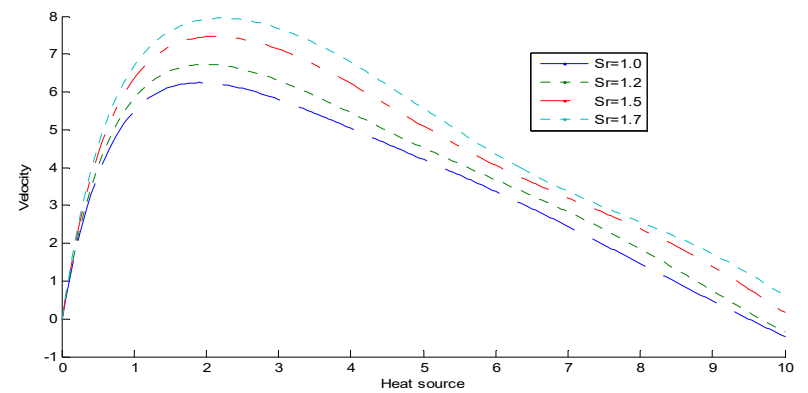

Fig. 4(b) Velocity $\left(u_{R}\right)$ versus Heat source parameter $(Q s)$ for arbitrary change in values of Soret number $(\mathrm{Sr})$.

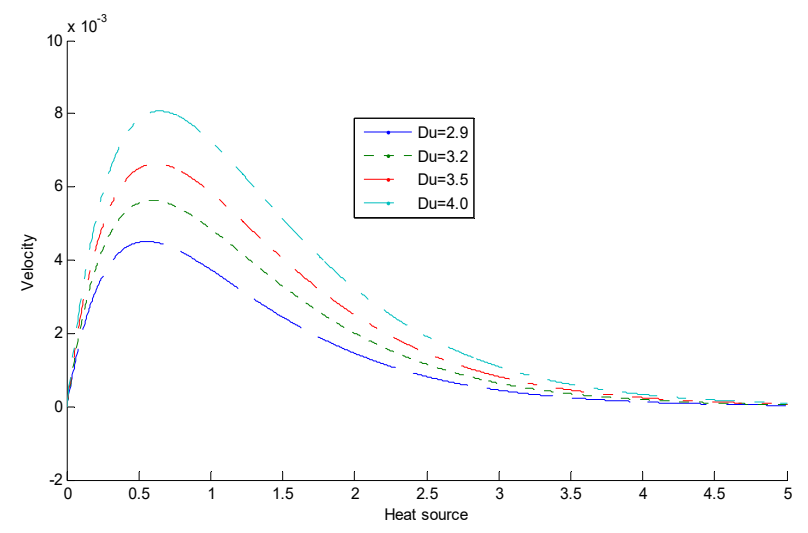

Fig. 4 (c) Velocity $\left(u_{R}\right)$ versus Heat source parameter $(Q s)$ for arbitrary change in values of Dufour number $(D u)$.

In Figure 5, the parametric effect of chemical reaction parameter $(F)$ and Hartmann number $(\mathrm{Ha})$ are demonstrated on the velocity profile $\left(u_{R}, y\right)$ for a set of fixed values of $D u=0.2, S r=1.0, Q s=0.2, \alpha=1.0476$, $G r=0.2$, and $G m=0.01$ (fig.5), $H a=3.0$ (fig.5(a)) and $F=1.0$ (fig.5(b)). It is observed from fig. 5(a) that, due to an increase in chemical reaction parameter $(F)$, the concentration of the fluid constituents is lowered (as observed in fig.3(d)), this results in minimizing the effect of mass bouncy force, which thus decelerates the flow rate against normal distance as such the velocity of flow slowed down. Figure 5(b) clearly shows that the fluid velocity decreases due to increases in Hartmann number (Ha) against normal distance. Due to increase in magnetic field parameter a resistive force in terms of Lorentz force generated within the flow domain, which acts against the flow as such decelerates the flow rate followed by decreasing the fluid velocity.

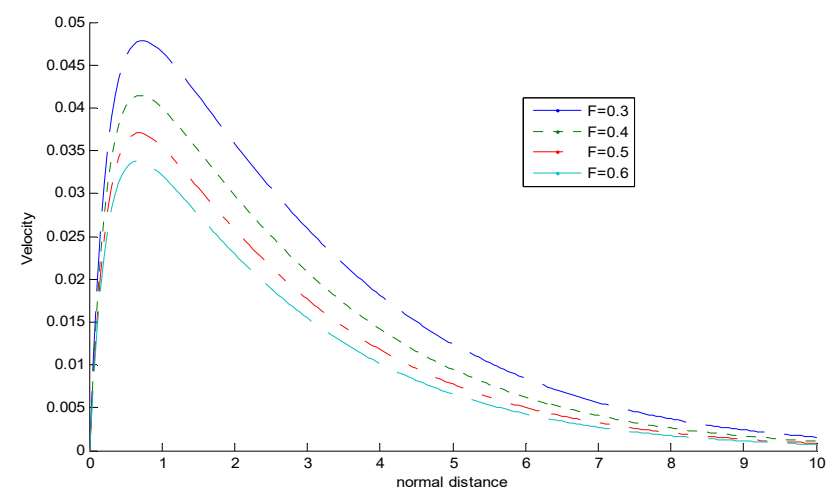

Fig. 5 (a) Velocity $\left(u_{R}\right)$ versus Normal distance (y) for arbitrary change in values of Chemical reaction parameter $(F)$.

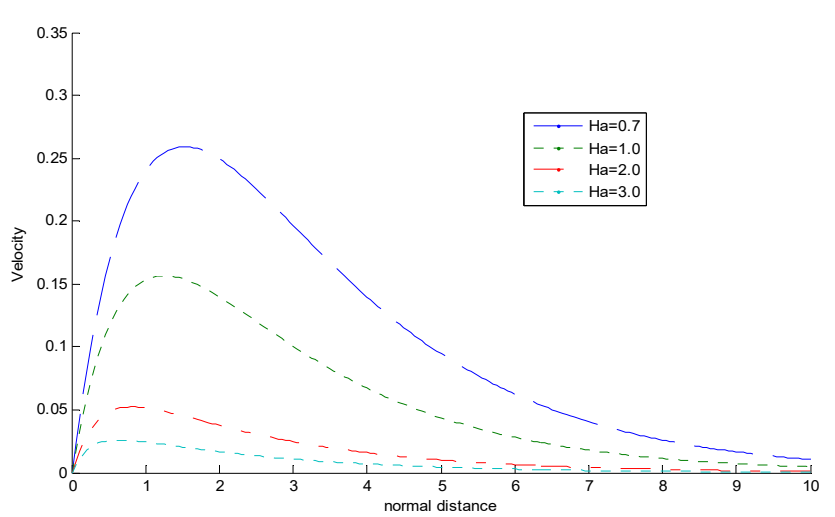

Fig. 5 (b) Velocity $\left(u_{R}\right)$ versus Normal distance (y) for arbitrary change in values of Hartmann number $(H a)$. 


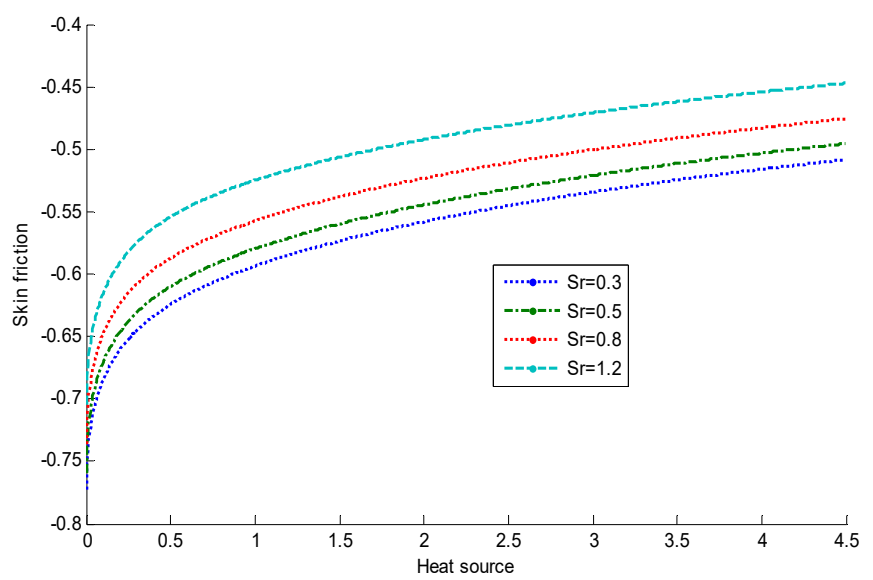

Fig. 6 (a) Skin-friction $\left(\tau_{R}\right)$ versus Heat source parameter $\left(Q_{S}\right)$ for arbitrary change in values of Soret number $(\mathrm{Sr})$.

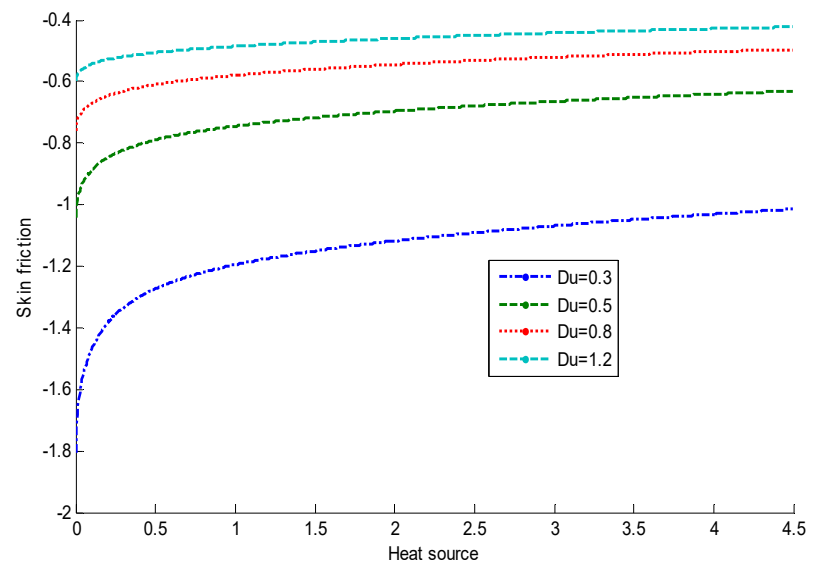

Fig. 6 (b) Skin-friction $\left(\tau_{R}\right)$ versus Heat source parameter $(Q s)$ for arbitrary change in values of Dufour number $(D u)$.

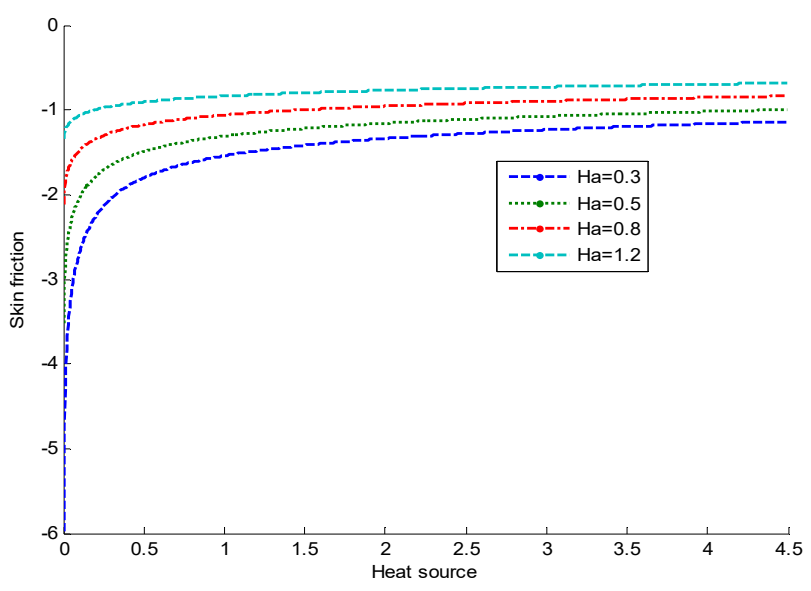

Fig.6 (c) Skin-friction $\left(\tau_{R}\right)$ versus Heat source parameter $(Q s)$ for arbitrary change in values of Hartmann number $(\mathrm{Ha})$.

The influence of Soret $(S r)$, Dufour $(D u)$ numbers and the Hartmann number $(\mathrm{Ha})$ on the skin-friction profile $\left(\tau_{R}, Q s\right)$ is depicted in fig. 6 for a set of fixed assigned value of $F=1.0, G r=1.0$, $G m=0.5, \alpha=1.0476$, (fig.6), $H a=2.0$, (fig.6(a) and 6(b)), $D u=0.3$ (fig. 6(a) and 6(c)), $S r=0.5$ (6(b) and 6(c)). As expected the skin-frictional effect raises due to increase in Soret $(S r)$ and Dufour $(D u)$ numbers.
The increase in the bouncy forces due to increase in Soret $(\mathrm{Sr})$ and Dufour $(D u)$ numbers are found to accelerate the flow rate as observed in fig. 4(b) and 4(c). As the skin-friction in Newtonian fluid directly proportional to the first power of the velocity gradient as such the acceleration of the flow rate helps in increasing the frictional effect of the fluid on the solid surface. Thus the value of skin-friction is found in increasing trend due to presence of Soret and Dufour numbers. On the other hand due to the presence of a magnetic field, a resistive force in terms of Lorentz force generates in the flow region which is being clubbed with the internal friction results of which the values of skinfriction are found increasing.

In Figure 7, the parametric effect of Hartmann number $(\mathrm{Ha})$, Soret number $(S r)$, Dufour number $(D u)$, heat source parameter $(Q s)$ and chemical reaction $(F)$ parameters on the induced magnetic field profile $\left(B_{x}, y\right)$ are depicted for fixed value of $G r=0.02, G m=0.02$ and $\alpha$ $=1.0476$ (fig.7), $F=2.0, Q s=1.5, D u=0.2$ (fig.7(a)), $F=1.0$ (fig.7(b), 7(c), 7(d)), $\mathrm{Ha}=3.0$ (fig.7(b), 7(c), 7(d), and 7(e)), Qs=0.2 fig.7(b), 7(c), and $7(\mathrm{e})), \mathrm{Sr}=0.5(7(\mathrm{c}), 7(\mathrm{~d})$, and $7(\mathrm{e}))$. It is clearly observed from these figures that, the induced magnetic field is found in increasing trend due to increase in values of Hartmann number $(\mathrm{Ha})$ and Soret number $(\mathrm{Sr})$, while a reverse trend is shown due to increase in the values of Dufour number $(D u)$ heat source parameter $(Q s)$ and chemical reaction parameter $(F)$. The enhancement in Hartmann number $(\mathrm{Ha})$ indicates the increasing strength of the applied magnetic field and as expected this increases the strength of induced magnetic field also. Due to increase in soret $(\mathrm{Sr})$ number, the concentration of the electrically conducting fluid particles increases which in turn increases the strength of the induced magnetic field. Again as the temperature of the electrically conducting fluid particles rises due to the presence of Dufour $(D u)$ number and heat source parameter $(Q s)$, the magnetization effect of the field gets diminish which thus decreases the strength of the induced magnetic field. Again as expected due to increases in chemical reaction parameter, the density of the electrically conducting fluid particles reduces. This reduction of charge particles near the solid surface is seen to lower the influence of applied as well as induced magnetic field.

Figure 8 Shows the parametric influence of Hartman number $(\mathrm{Ha})$ and magnetic field inclination parameter $(\alpha)$ to the total magnetic field profile $(B, y)$ for a set of fixed value of $F=1.0, Q s=0.02, D u=0.01$, $H a=3.0$, (fig.8(a)) $S r=1.5, B_{0}=0.5, G r=0.02, G m=0.02$. ( Fig. 8 (a) and $8(\mathrm{~b})), F=2.0, Q s=1.5, D u=0.2, \alpha=1.0476$. As expected due to increase in values of magnetic field inclination parameter as well as Hartmann number, the effect of total magnetic field diminishes.

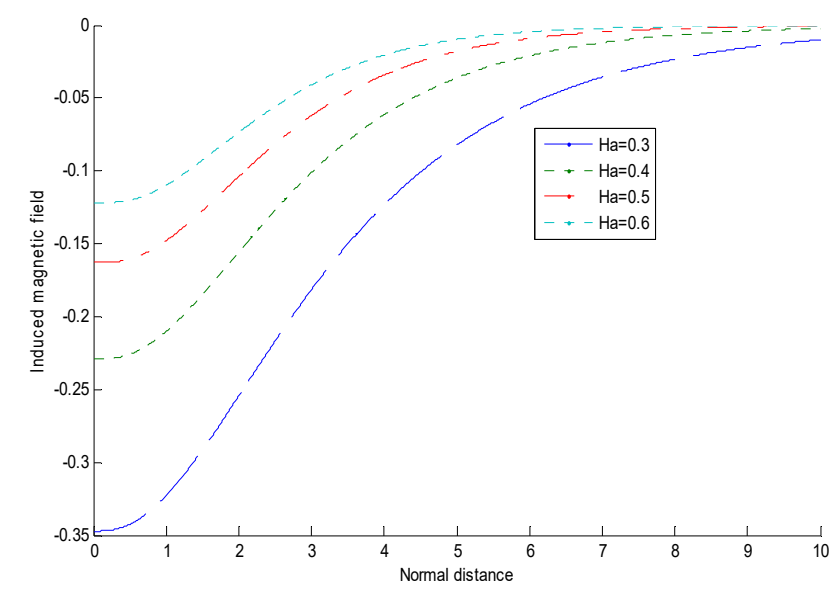

Fig. 7 (a) Induced magnetic field ( $B_{x}$ ) versus normal distance $(y)$ for arbitrary change in values of Hartmann number $(H a)$. 


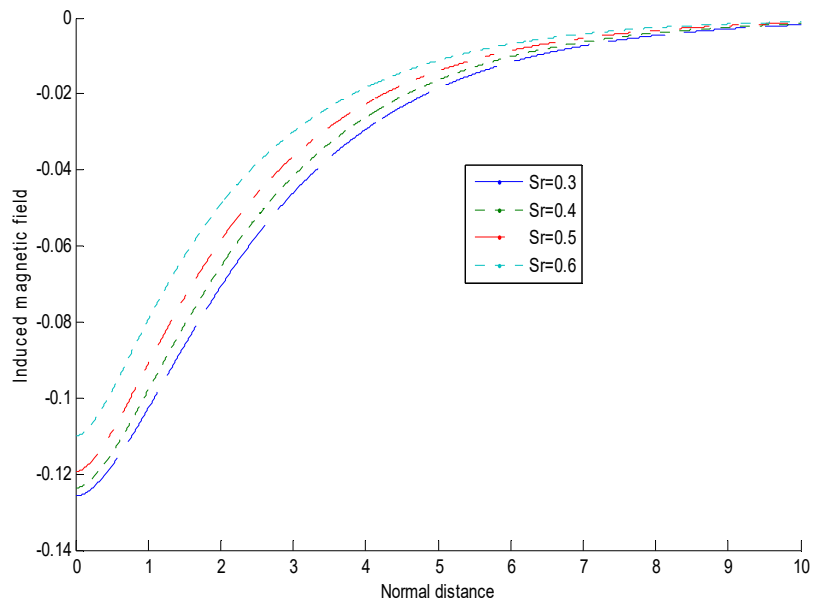

Fig. 7 (b) Induced magnetic field ( $B_{x}$ ) versus normal distance $(y)$ for arbitrary change in values of Soret number $(\mathrm{Sr})$.

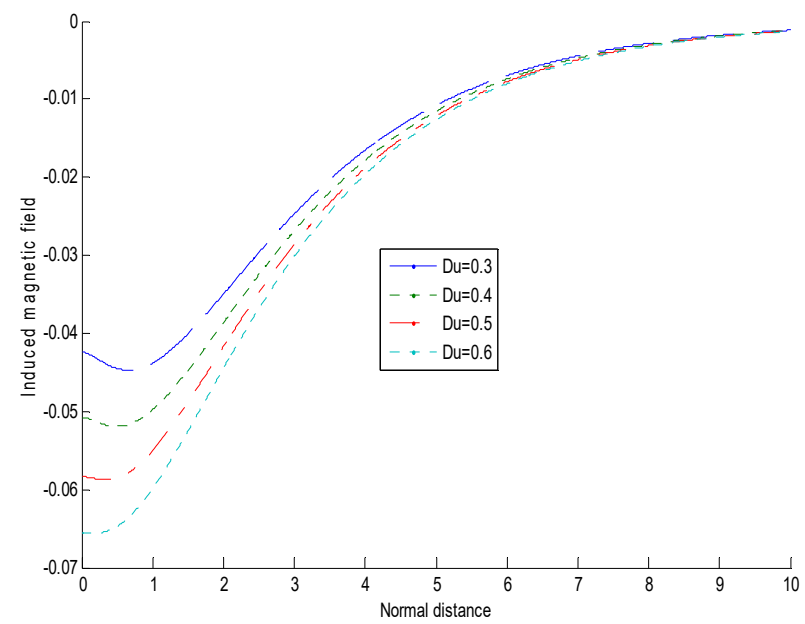

Fig. 7 (c) Induced magnetic field ( $B_{x}$ ) versus normal distance $(y)$ for arbitrary change in values of Dufour number $(D u)$.

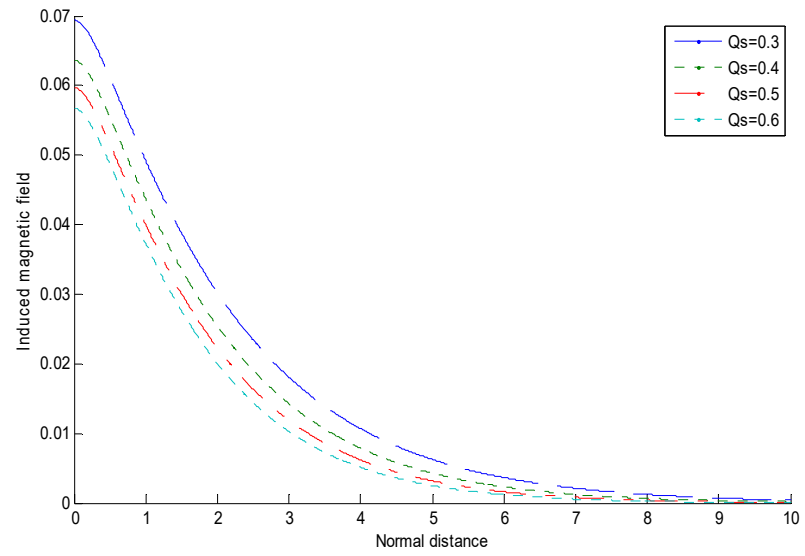

Fig. 7 (d) Induced magnetic field ( $B_{x}$ ) versus normal distance $(y)$ for arbitrary change in values of Heat source parameter $\left(Q_{s}\right)$.

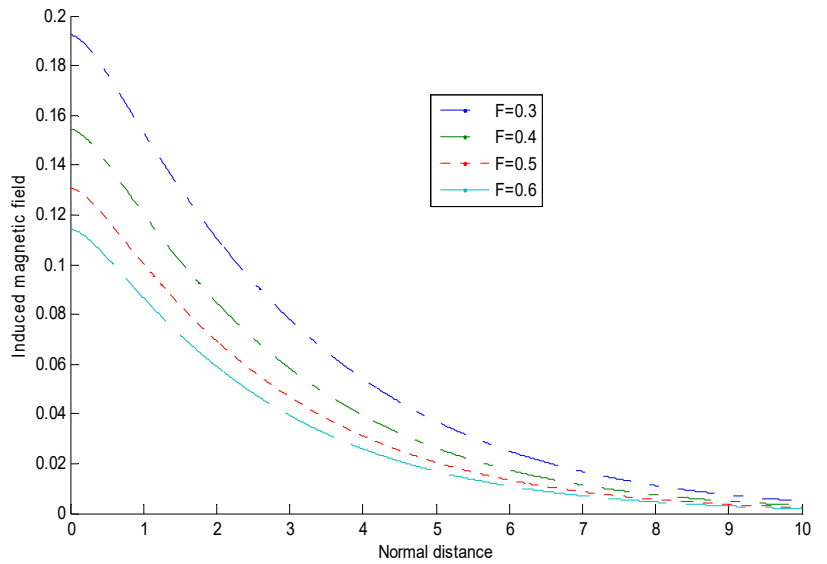

Fig. 7 (e) Induced magnetic field ( $B_{x}$ ) versus normal distance $(y)$ for arbitrary change in values of Chemical reaction parameter $(F)$.

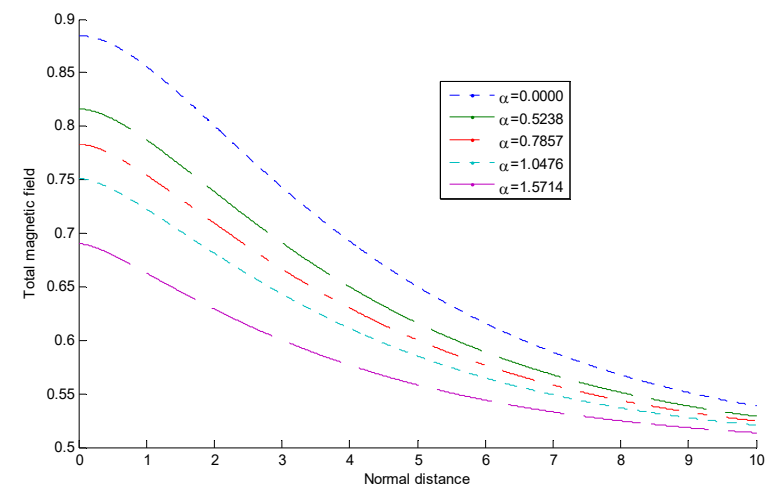

Fig. 8 (a) Total magnetic field ( $\vec{B}$ ) versus normal distance $(y)$ for arbitrary change in values of inclination $(\alpha)$.

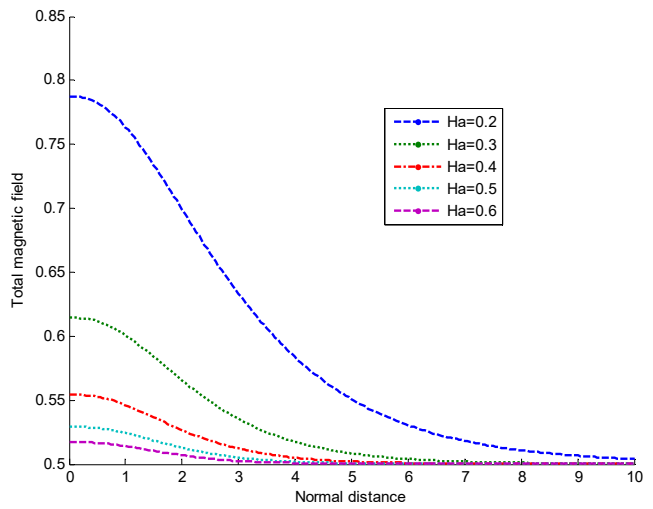

Fig. 8 (b) Total magnetic field $(B)$ versus normal distance $(y)$ for arbitrary change in values of Hartmann number $(\mathrm{Ha})$.

In figure 9, the parametric effect of Hartmann number $(\mathrm{Ha})$ and magnetic field inclination parameter $(\alpha)$ on the current density profile $(J, y)$ is depicted for a set of fixed values of $F=1.0, Q_{s}=0.2, D u=0.02$, $S r=0.5, \alpha=1.0476, G r=0.02, G m=0.02, \alpha=1.0476$ (fig.9 (a) only) and $H a=3.0$ (fig.9(b) only). It is observed that, the current density is decreasing with the enhancement of both Hartmann number and magnetic field inclination parameter. It is also observed that in presence of Hartman number, the current density achieves its maximum peak at $\mathrm{y}=2.5$ (approx) and thereafter gradually decreases far away from the plate, while in accompany with magnetic inclination parameter, the current density attains the minimum peak at $y=1.0$ (approx) and thereafter gradually increases towards the free stream region. 


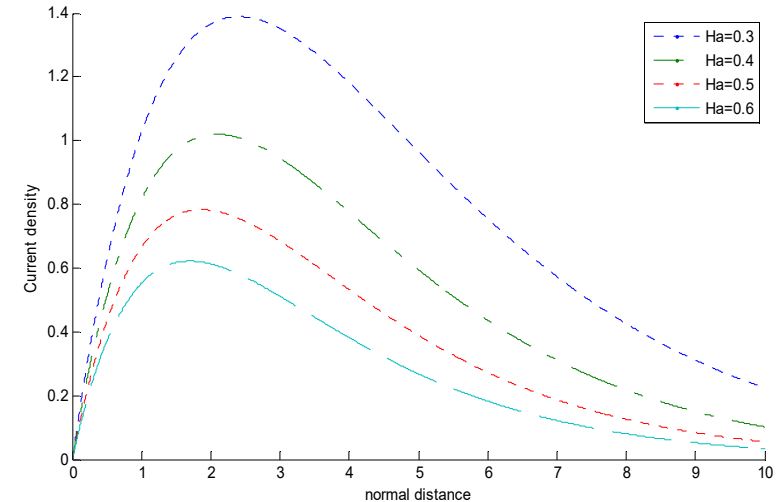

Fig. 9 (a) Current density $(J)$ versus Normal distance $(y)$ for arbitrary change in values of Hartmann number $(\mathrm{Ha})$.

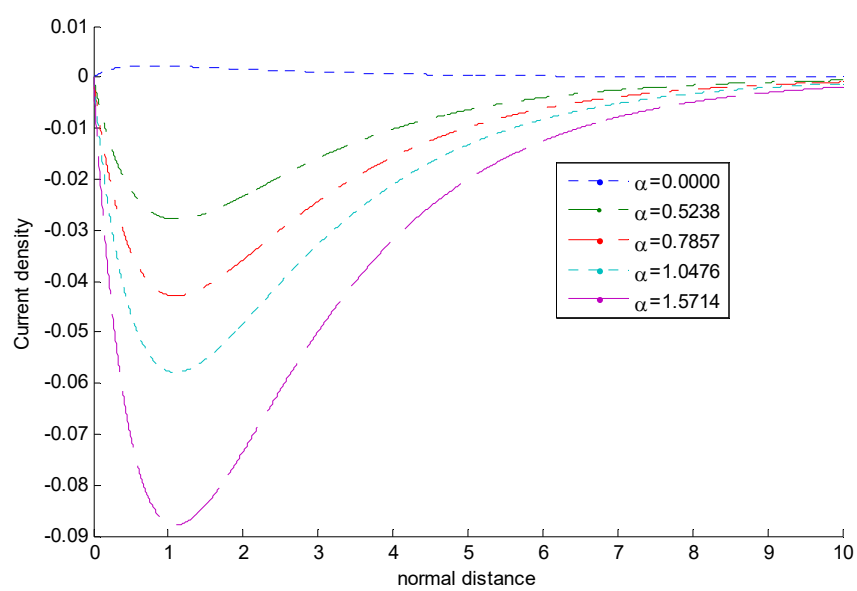

Fig. 9 (b) Current density ( $J$ ) versus Normal distance (y) for arbitrary change in values of magnetic field inclination parameter $(\alpha)$

Table 1 demonstrates the influence of Dufour $(D u)$ number on the temperature profile against heat source parameter $(Q s)$ for a set of fixed values of $F=1.2, S r=1.8$. Table 1 clearly shows that, the temperature of

the fluid particles gradually takes the upper trends due to increase in Dufour number $(D u)$ against the heat source parameter. Table 2 shows a comparative data wise variations in the values of Nusselt number $\left(N u_{R}\right)$ and Sherwood number $\left(S h_{R}\right)$ against heat source parameter $(Q s)$ for arbitrary change in values of Dufour $(D u)$ number and for a set of fixed values of $F=1.0, S r=1.8$. Due to increase in Dufour $(D u)$ number, the heat transfer as well as mass transfer rates from the plate surface (higher temperature zone /concentration zone) to the free stream region (lower temperature zone /concentration zone) accelerate which in turn increases the value of Nusselt number and Sherwood number respectively. On the other hand a complementary effect on the rates of heat and mass transfer phenomena are observed for $Q s \in[1.0,4.0]$. The numerical variation in the values of Nusselt number as well as Sherwood number for arbitrary variations in Soret number $(\mathrm{Sr})$ against heat source parameter $(Q s)$ is depicted in table 3 , for a set of preassigned values of $F=1.0$ and for $D u=3.7$, (Nusselt number $\left.\left(N u_{R}\right)\right)$ and for $D u=1.1$ ( Sherwood number $\left(S h_{R}\right)$ ). The influence of Soret number on the rates of heat and mass transfer phenomena is seen to accelerate the transport rates as such both the Nussult number and the Sherwood number are found to be increased. It is interesting to observed that due to increase in heat source parameters $(Q s)$, Nusselt number shown an increasing trend, while Sherwoood number shown a reversible trend.

Table 1Showing the numerical values of temperature $\left(\theta_{R}\right)$ against Heat source parameter $(Q s)$ for arbitrary variation of Dufour number $(\mathrm{Du})$,

\begin{tabular}{|l|l|l|l|l|}
\hline$Q_{s}$ & $D u=1.2$ & $D u=1.3$ & $D u=1.4$ & $D u=1.5$ \\
\hline 0.1 & 0.00876 & 0.01350 & 0.01594 & 0.01652 \\
\hline 0.2 & 0.03815 & 0.04738 & 0.05420 & 0.05907 \\
\hline 0.3 & 0.05635 & 0.06849 & 0.07810 & 0.08567 \\
\hline 0.4 & 0.06944 & 0.08380 & 0.09551 & 0.10508 \\
\hline 0.5 & 0.07957 & 0.09578 & 0.10920 & 0.12038 \\
\hline 0.6 & 0.08776 & 0.10558 & 0.12049 & 0.13305 \\
\hline 0.7 & 0.09458 & 0.11387 & 0.13010 & 0.14388 \\
\hline 0.8 & 0.10038 & 0.12104 & 0.13849 & 0.15337 \\
\hline 0.9 & 0.10538 & 0.12735 & 0.14594 & 0.16185 \\
\hline
\end{tabular}

Table 2 Showing the numerical values of Nusselt number $\left(N u_{R}\right)$, Sherwood number $\left(S h_{R}\right)$ against heat source parameter $\left(Q_{s}\right)$ for arbitrary variation of Dufour number $(D u)$.

\begin{tabular}{|c|c|c|c|c|c|c|c|c|}
\hline \multirow{2}{*}{$Q s$} & \multicolumn{4}{|c|}{$N u_{R}$} & \multicolumn{4}{|c|}{$S h_{R}$} \\
\hline & $D u=1.3$ & $D u=1.4$ & $D u=1.5$ & $D u=1.6$ & $D u=1.3$ & $D u=1.4$ & $D u=1.5$ & $D u=1.6$ \\
\hline 1.0 & 0.24496 & 0.25710 & 0.26563 & 0.27162 & 0.31040 & 0.32596 & 0.34137 & 0.35671 \\
\hline 1.5 & 0.34532 & 0.35460 & 0.36051 & 0.36413 & 0.19554 & 0.20573 & 0.21565 & 0.22534 \\
\hline 2.0 & 0.41382 & 0.42230 & 0.42727 & 0.42989 & 0.13821 & 0.14662 & 0.15474 & 0.16262 \\
\hline 2.5 & 0.46284 & 0.47188 & 0.47701 & 0.47954 & 0.10181 & 0.10928 & 0.11647 & 0.12341 \\
\hline 3.0 & 0.49788 & 0.50866 & 0.51483 & 0.51797 & 0.07584 & 0.08271 & 0.08931 & 0.09567 \\
\hline 3.5 & 0.52174 & 0.53540 & 0.54345 & 0.54783 & 0.05599 & 0.06243 & 0.06862 & 0.07457 \\
\hline 4.0 & 0.53587 & 0.55367 & 0.56444 & 0.57070 & 0.04011 & 0.04623 & 0.05210 & 0.05775 \\
\hline
\end{tabular}

Table 3 Showing the numerical values of Nusselt number $\left(N u_{R}\right)$ and Sherwood number $\left(S h_{R}\right)$ against heat source parameter $\left(Q_{s}\right)$ for arbitrary variation of Soret number $(\mathrm{Sr})$.

\begin{tabular}{|c|c|c|c|c|c|c|c|c|}
\hline \multirow{2}{*}{$Q s$} & \multicolumn{4}{|c|}{$N u_{R}$} & \multicolumn{4}{|c|}{$S h_{R}$} \\
\hline & $S r=1.3$ & $S r=1.4$ & $S r=1.5$ & $S r=1.6$ & $S r=1.3$ & $S r=1.4$ & $S r=1.5$ & $S r=1.6$ \\
\hline 1.0 & 0.12473 & 0.22333 & 0.23602 & 0.24716 & 0.25912 & 0.26853 & 0.27307 & 0.27566 \\
\hline 1.5 & 0.20879 & 0.29063 & 0.30343 & 0.31455 & 0.12649 & 0.13953 & 0.15040 & 0.15957 \\
\hline 2.0 & 0.27580 & 0.34174 & 0.35466 & 0.36579 & 0.05789 & 0.07473 & 0.08892 & 0.10101 \\
\hline 2.5 & 0.32665 & 0.38302 & 0.39609 & 0.40727 & 0.01537 & 0.03424 & 0.05022 & 0.06388 \\
\hline 3.0 & 0.36764 & 0.41753 & 0.43081 & 0.44210 & -0.01446 & 0.00569 & 0.02279 & 0.03744 \\
\hline 3.5 & 0.40183 & 0.44703 & 0.46056 & 0.47201 & -0.03700 & -0.01596 & 0.00192 & 0.01727 \\
\hline 4.0 & 0.43093 & 0.47260 & 0.48644 & 0.49810 & -0.05488 & -0.03318 & -0.01471 & 0.00116 \\
\hline
\end{tabular}




\section{CONCLUSIONS}

A theoretical study on the steady, free convictive heat and mass transfer magnetohydrodynamic flow of an incompressible, viscous fluid in presence of thermo-diffusion (Soret) and diffusion-thermo (Dufour) effects along with a first order chemical reaction is analyzed. The influence of induced magnetic field is taken in to account, while the effect of induced electric field is neglected due to weak polarization of charges. The parametric influences of some dimensionless thermo physical as well as magneto - physical parameters on the nondimensional velocity field, induced magnetic field, temperature field, concentration field and on wall heat and mass transfer as well as on skin-friction, induced and total magnetic fields and on the current density are investigated graphically and in tabular forms. Some of the significant outcomes of the study is as follows.

7. The temperature field increases due to increase in heat source parameter as well as Dufour number however the temperature decreases due to increase in chemical reaction parameter.

8. The concentration field decreases due to increase in heat source parameter and chemical reaction parameter but increases with Soret and Dufour numbers.

9. The velocity field and induced magnetic field increases with an increase in Soret number where as they decrease due to increases in chemical reaction parameter.

10. The skin-friction and induced magnetic field increase due to increase in Hartmann number, while induced magnetic field decreases with the increase in values of heat source parameter.

11. The total magnetic field decreases due to increase in magnetic field inclination parameter.

12. The Nusselt number as well as Sherwood number takes the upper trends due to increase in Soret and Dufour numbers while a complimentary effect is observed due to increase in heat source parameter.

\section{NOMENCLATURE}

$B_{0} \quad$ Strength of the applied magnetic field

$\vec{B} \quad$ Total magnetic field

$B_{x} \quad$ Induced magnetic field

$C_{p} \quad$ Specific heat at constant pressure

$C^{*} \quad$ Species concentration

$C_{s} \quad$ Susceptibility concentration

$C_{w}^{*} \quad$ Wall Species concentration (Dimensional)

$C_{\infty}^{*} \quad$ Species concentration in the free stream

$D_{M} \quad$ Mass diffusivity co-efficient

Du Dufour number

$F \quad$ Chemical reaction parameter

Gm Grashof number for mass transfer

$\mathrm{Gr} \quad$ Grashof number for heat transfer

$G \quad$ Acceleration due to gravity

$J_{z}^{*} \quad$ Current density

K Thermal conductivity

$k_{1} \quad$ Mean absorption co-efficient

$k_{l}$

$K_{T}$

$M$

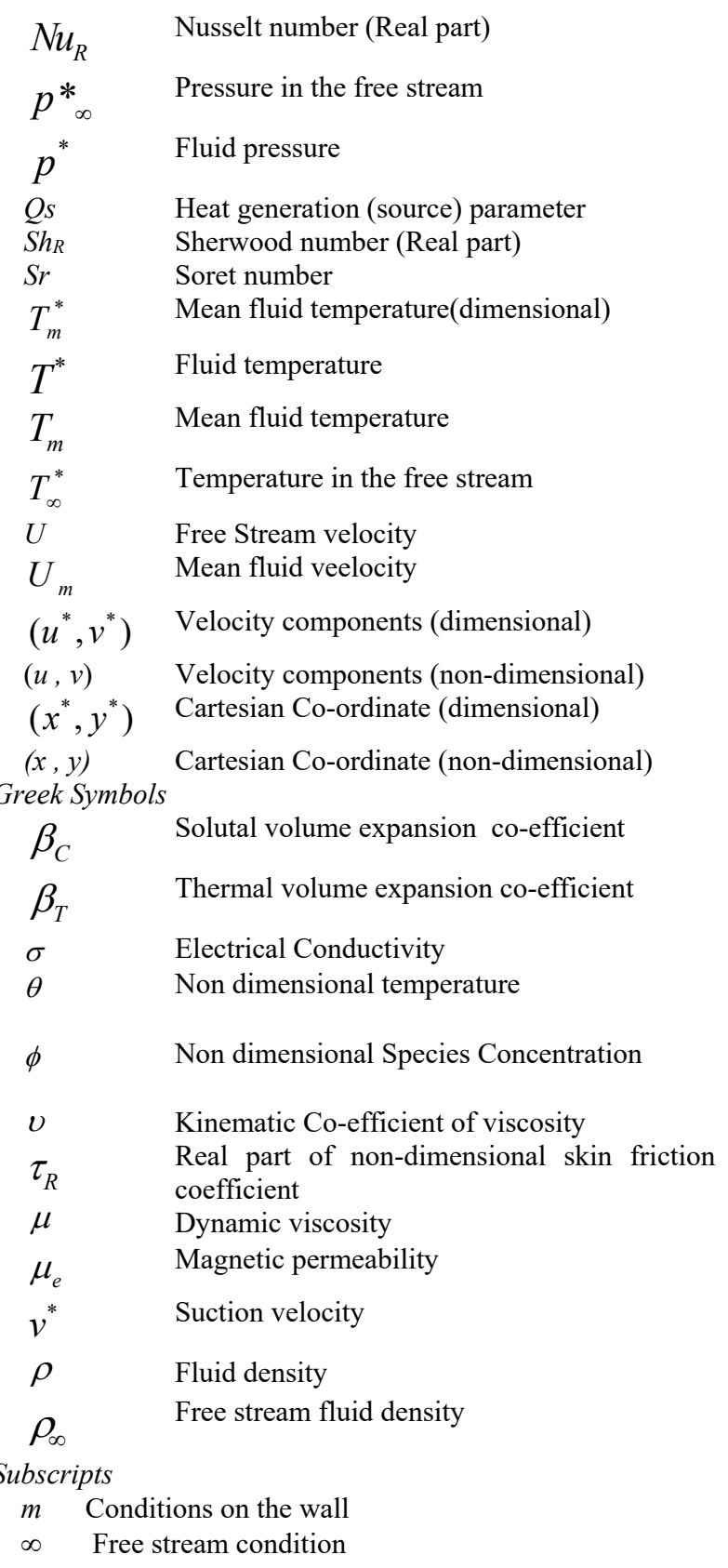

\section{REFERENCES}

Ahmed, N., Sengupta, S., and Datta, D., 2013, “An Exact Analysis for MHD Free Convection Mass Transfer Flow Past an Oscillating Plate Embedded in a Porous Medium with Soret Effect," Chemical Engineering Communications. 200 (4), 494-513. https://doi.org/10.1080/00986445.2012.709474

Ahmed, S., 2010, "Induced Magnetic Field with Radiation Fluid Over a Porous Vertical Plate: Analytical Study," Journal of Naval Architecture and Marine Engineering.7, 83-94.

http://dx.doi.org/10.3329/jname.v7i2.5662

Ahmed, S., Zueco, J., Luis, M., and González, L., 2017, "Effects of Chemical Reaction, Heat and Mass Transfer and Viscous Dissipation Over a MHD Flow in a Vertical Porous Wall Using Perturbation Method," International Journal of Heat and Mass Transfer.104, 409418.

https://doi.org/10.1016/j.ijheatmasstransfer.2016.07.076 
Akbar, N. S., Raza, M., and Ellahi, R., 2015, "Influence of Induced Magnetic Field and Heat Flux with the Suspension of Carbon Nano Tubes for the Peristaltic Flow in a Permeable Channel," Journal of magnetism and magnetic materials.381,405-415.

https://doi.org/10.1016/j.jmmm.2014.12.087

Anghel, M., Takhar, H. S., and Pop, I., 2000, "Dufour and Soret Effects on Free Convection Boundary Layer Flow over a Vertical Surface Embedded in a Porous Medium," Studia universities. Babes Bolyai Mathematica.45 (4), 11-21.

Anwar Beg, O., Bekier, A. Y., Prasad, V. R., Zueco, J., and Ghosh, S. K., 2009, "Non-Similar, Laminar, Steady, Electrically Conducting Forced Convection Liquid Metal Boundary Layer Flow with Induced Magnetic Field Effects," International Journal of Thermal Sciences. 48(8), 1596-1606.

https://doi.org/10.1016/j.ijthermalsci.2008.12.007

Bhavana, M., ChennaKesavaiah, D., and Sudhakaraiah, A., 2013, "The Soret Effect on Free Convective Unsteady MHD Flow over a Vertical Plate with Heat Source," International Journal of Innovative Research in Science, Engineering and Technology.2 (5), 1617-1628.ISSN: 23198753

Chaudhary, R. C., and Sharma, B. K., 2006, "Combined Heat and Mass Transfer by Laminar Mixed Convection Flow from a Vertical Surface with Induced Magnetic Field,"Journal of Applied Physics. 99(3), 034901-034910.

https://doi.org/10.1063/1.2161817

Denno, K. I., 1972, "Effect of Induced Magnetic Field on the Magnetohydrodynamic Channel Flow," IEEE Trans. Electron. Devices. 19 (3), 322-331.

http://dx.doi.org/10.1109/T-ED.1972.17421

Eckert, E. R. G., and Drake, R. M., 1972, "Analysis of Heat and Mass Transfer," Hemisphere Pub. Corp. Washington, D.C.

EI-dabe Nabil, T., Ghaly Ahmed, Y., RizkallahRaafat, R., EwisKarem, M., and AmeenAL-bareda, M., 2015, "Comparison Between Numerical and Analytical Solution for the Motion of Viscous Fluid with Heat and Mass Transfer Through Porous Medium Over a Vertical Infinite Permeable Plate in the Presence of Induced Magnetic Field," International Journal of Current Engineering and Technology. 5(3), 1890-1897.E-ISSN 2277 - 4106, P-ISSN 2347 - 5161

Ghosh, S. K., Beg, O. A., and Zueco, J., 2010, "Hydromagnetic Free Convection Flow with Induced Magnetic Field Effects,"Meccanica.14, 175-185.

http://dx.doi.org/ 10.1007/s11012-009-9235-x

Haque, M. M., and Alam, M. M., 2009, "Transient Heat and Mass Transfer by Mixed Convection Flow from a Vertical Porous Plate with Induced Magnetic Field, Constant Heat and Mass Fluxes," Mechanics and Thermics (Journal of AMSE). 78(4), 54-73.

Jha, B. K., and Sani, I., 2013, "Computational Treatment of MHD of Transient Natural Convection Flow in a Vertical Channel due to Symmetric Heating in Presence of Induced Magnetic Field," Journal of the physical society of Japan.82(8), 084401-084409.

https://doi.org/10.7566/JPSJ.82.084401

Kumar, A., and Singh, A. K., 2013, "Unsteady MHD Free Convective Flow Past a Semi-Infinite Vertical Wall with Induced Magnetic Field," Applied Mathematics and Computation.222, 462-471.

https://doi.org/10.1016/j.amc.2013.07.044
Kwanza, J. K., and Balakiyema, J. A., 2012, "Magnetohydro-Dynamic Free Convective Flow Past an Infinite Vertical Porous Plate with Magnetic Induction,” Journal of Fusion Energy. 31 (4), 352-356.

http://x.doi.org/10.1007/s10894-011-9475-3

Mamatha, B., Raju, M. C., and Varma, S. V. K., 2015, "Thermal Diffusion Effect on MHD Mixed Convection Unsteady Flow of a Micro Polar Fluid Past a Semi-Infinite Vertical Porous Plate with Radiation and Mass Transfer," International Journal of Engineering Research in Africa.13, 21-37.

http://dx.doi.org/10.4028/www.scientific.net/JERA.13.21

Muthtamilselvan, M., Prakash, D., and Doh, D. H., 2014, "Effect of Non-Uniform Heat Generation on Unsteady MHD Flow Over a Vertical Stretching Surface with Variable Thermal Conductivity," Cambridge Journal of Mechanics.30 (02), 199-208. https://doi.org/10.1017/jmech.2013.71

Platten, J. K., and Legros, J. C., 1984, "Convection in Liquids," Springer-Verlag. ISBN, 0387126376.

Postelnicu, A., 2004, "Influence of a Magnetic Field on Heat and Mass Transfer by Natural Convection from Vertical Surfaces in Porous Medium Considering Soret and Dufour Effects," International Journal of heat and mass transfer.47(6-7), 1467-1472.

https://doi.org/10.1016/j.ijheatmasstransfer.2003.09.017

Raju, C. S. K., Sandeep, N., Sulochana, C. V., Sugunamma, V., Jayachandra Babu M., 2015,"Radiation, Inclined Magnetic Field and Cross-Diffusion Effects on Flow Over a Stretching Surface," Journal of the Nigerian Mathematical Society.34,169-180.

https://doi.org/10.1016/i.jnnms.2015.02.003

Raju, M. C., Varma, S. V. K., Reddy, P. V., and Saha, S., 2008, "Soret Effects due to Natural Convection Between Heated Inclined Plates with Magnetic Field," Journal of Mechanical Engineering.39(2), 43-48. http://dx.doi.org/10.1.1.429.6841

Reddy, C. P., Raju, M. C., and Raju, G. S. S., 2016, "Soret and Dufour Effects on MHD Free Convection Flow of Rivlin-Ericksen Fluid Past a Semi-Infinite Vertical Plate," Advances and Applications in Fluid Mechanics.19 (2), 401- 414.

http://dx.doi.org/10.17654/FM019020401

Sajid M., Ali N. Abbas Z.and Javed, T., 2015, "On Modeling of TwoDimensional MHD Flow with Induced Magnetic Field: Solution of Peristaltic Flow of a Couple Stress Fluid in a Channel" Iranian Journal of Science \& Technology.39 (A1), 35-43.

http://dx.doi.org/10.22099/IJSTS.2015.2888

Sandeep, N., and Sugunamma, V., 2014, "Radiation and Inclined Magnetic Field Effects on Unsteady Hydromagnetic Free Convection Flow Past an Impulsively Moving Vertical Plate in a Porous Medium," Journal of Applied Fluid Mechanics.7 (2), 275-286.ISSN 1735-3572, EISSN 1735-3645.

Sarveshanand, and Singh, A. K., 2015, "Magnetohydro-Dynamic Free Convection between Vertical Parallel Porous Plates in the Presence of Induced Magnetic Field," Springer Plus. 4 (333), 1-13. http://dx.doi.org/10.1186/s40064-015-1097-1

Sengupta, S., 2015, “An Analysis on Unsteady Heat and Mass Transfer Flow of Radiative Chemically Reactive Fluid Past an Oscillating Plate Embedded in Porous Media in Presence of Soret Effect," International Journal of Recent Technology and Engineering.3(6), 10-14.ISSN: 2277-3878 
Sengupta, S., and Ahmed, N., 2015, "Chemical Reaction Interaction on Unsteady MHD Free Convective Radiative Flow Past an Oscillating Plate Embedded in Porous Media with Thermal Diffusion," Advances in Applied Science Research.6(7), 87-104.ISSN: 0976-8610 CODEN (USA): AASRFC

Sengupta, S., and Ahmed, N., 2016, "MHD Free Convective Mass Transfer Flow of Radiative Uniform Heat Generation (Absorption) Fluid through a Wavy Permeable Channel in Presence of Soret and Dufour Effects," Canadian Journal of Physics.95 (1), 44-58. https://doi.org/10.1139/cjp-2014-0599

Sengupta, S., and Karmakar, A., 2016, "MHD Mixed Convection Chemically Reactive Flow in Radiative Heat Generating Medium with Soret Effect," International Journal of Modern Mathematical Sciences. 14 (3), 239-261.ISSN: 2166-286X

Sengupta, S., and Sen, M., 2013, "Free Convective Heat and Mass Transfer Flow Past an Oscillating Plate with Heat Generation, Thermal Radiation and Thermo Diffusion Effects," J. P. J. of heat and mass transfer. 8 (2), 187-210.

Singh, N. K., Kumar, V., and Sharma, G. K., 2016, "The Effect of Inclined Magnetic Field on Unsteady Flow Past on Moving Vertical Plate with Variable Temperature,"IJLTEMAS. 5(2), 34-37.ISSN 2278 2540

Singh, R. K., Singh, A. K., Sacheti, N. C., and Chandran, P., 2010, “On Hydromagnetic Free Convection in the Presence of Induced Magnetic Field," Heat Mass Transf.46 (5), 523-529. https://doi.org/10.1007/s00231-010-0594-6

Soundalgekar, V. M., 1965, "Hydro Magnetic Flow Near an Accelerated Plate in the Presence of a Magnetic Field," Applied Scientific Research. 12(1), 151-156.

\section{https://doi.org/10.1007/BF02923392}

Sravanthi, C. S., LeelaRatnam, A., and Bhaskar Reddy, N., 2013, "Thermo-Diffusion and Chemical Reaction Effects on a Steady Mixed Convective Heat and Mass Transfer Flow with Induced Magnetic Field," International Journal of Innovative Research in Science, Engineering and Technology. 2(9), 4415-4424.ISSN: 2319-8753

Srinivasacharya, D., Mallikarjuna, B., and Bhuvanavijaya, R., 2015, "Soret and Dufour Effects on Mixed Convection along a Vertical Wavy Surface in a Porous Medium with Variable Properties," Ain Shams Engineering Journal. 6(2), 553-564. https://doi.org/10.1016/j.asej.2014.11.007

SudheerBabu, M., and Satya Narayana, P. V., 2009, "Effects of the Chemical Reaction and Radiation Absorption on Free Convection Flow Through Porous Medium with Variable Suction in the Presence of Uniform Magnetic Field," J.P. Journal of Heat and Mass Transfer.3,219-234.

Sugunamma, V., Sandeep, N., Krishna, P. M., and Bahunadam, R., 2013, "Inclined Magnetic Field and Chemical Reaction Effects on Flow Over a Semi-Infinite Vertical Porous Plate through Porous Medium," Communications in Applied Sciences. 1(1), 1-24.

http://dx.doi.org/10.1.1.899.2325

Venkataramana, B. S., Jalaja, P., and Jayakumar, R. K., 2017, "The Influence of Heat Generation (Absorption) and Thermal Radiation on MHD Laminar Boundary Layer Flow over a Moving Cylindrical Rod," International Journal of engineering Sciences and Research Technology. 6(2), 471-475.

http://dx.doi.org/10.5281/zenodo.293743 